Conclusion It is pertinent to point out the role of counseling, both pretest and posttest, in the National AIDS Control Program, India. ICTC provides comprehensive services, for identification and documentation of HIV related co-infections including TB and STIs. Very few studies have been conducted in our country analysing the outcome of linkage between National programs (NACP-IV, RNTCP and National STD control programs). Our centre took the initiative to study this linkage to aid in better patient outcome.

Disclosure No significant relationships.

\section{P246 COMMUNITY PERCEPTIONS, EXPERIENCES AND PREFERENCES FOR PARTNER NOTIFICATION SERVICES IN NORTH CAROLINA}

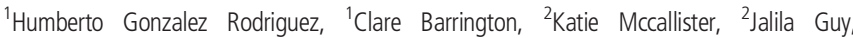
${ }^{2}$ Lisa Hightow-Weidman, ${ }^{3}$ Erika Samoff, ${ }^{3}$ Victoria Mobley, ${ }^{2}$ Arlene Seña* . ' UNC-CH Gillings School of Global Public Health, Health Behavior, Chapel Hill, USA; ${ }^{2}$ University of North Carolina at Chapel Hill, Division of Infectious Diseases, Chapel Hill, USA; ${ }^{3}$ North Carolina Division of Public Health, Communicable Disease Branch, Raleigh, USA

10.1136/sextrans-2019-sti.379

Background Men who have sex with men (MSM) and transgender women (TGW) have increased risk for syphilis and HIV. Partner notification (PN) is an effective strategy to provide early diagnosis and improve treatment outcomes among sexual partners of individuals diagnosed with an STI. We conducted a qualitative study to examine perceptions, experiences, and preferences for PN among Black and Latino MSM and TGW in North Carolina (NC).

Methods We conducted seven focus groups (FG) with 50 purposively sampled participants across four NC counties with high rates of HIV and syphilis. Eligible participants were aged 18-44, identified as Black and/or Latino, had ever had sex with men, and identified as male or a TGW. We used a semistructured guide to facilitate five groups in English and two in Spanish. We inductively analyzed data after each FG via field notes and team debriefs, organizing field notes in a matrix to identify crosscutting themes, and double-coding transcripts to systematically examine differences and similarities across salient themes.

Results Black males reported more familiarity and experiences with PN than Latino males and Black and Latino TGW. Generally, participants familiar with PN perceived the approach to locating clients as aggressive, while participants unfamiliar with $\mathrm{PN}$ viewed its theoretical application positively. All participants worried about personal privacy and stigma, on the part of PN staff or others. Poor communication and feeling harassed by staff during PN characterized negative experiences while empathy, privacy, choice and autonomy, and support with navigating services characterized positive experiences. Participants preferred using PN to notify casual partners but worried PN could antagonize relationships with steady partners.

Conclusion Participants prefer more choice, support and sensitivity in $\mathrm{PN}$. The systematic integration of shared-decision making and service navigation into PN could transform community perceptions and improve the quality and success of PN.

Disclosure No significant relationships.

\section{P247 \\ PARTNER NOTIFICATION FOR SYPHILIS IN CHILE: REALITIES FROM TWO REGIONAL HEALTH SERVICES - A QUALITATIVE CASE STUDY}

${ }^{1}$ Nicole Iturrieta-Guaita, ${ }^{1}$ Meredith Temple-Smith, ${ }^{2}$ Jane Tomnay*. ${ }^{1}$ The University of Melbourne, General Practice, Melbourne, Australia; ${ }^{2}$ The University of Melbourne, Department of Rural Health, Shepparton, Australia

\subsection{6/sextrans-2019-sti.380}

Background Partner Notification (PN) has long been considered essential for sexually transmissible infection (STI) control, but both policies and methods of implementation vary between countries. Although the provision of sexual health services has improved in Chile, syphilis is one of the most commonly reported STIs. This is the first study to explore the role of PN in syphilis control in public health services in Chile.

Methods A qualitative multiple case study was designed, using two regional Health Services as cases. Different data collection methods were used: a qualitative document analysis, a syphilis management clinic review in 20 health services, and semistructured interviews with 48 healthcare providers (HCPs) and $10 \mathrm{key}$ informants. The data was analysed using an inductive approach, with both within-case and cross-case thematic analysis. Selected quotes were translated from Spanish-English.

Results Findings revealed that syphilis management has a wellorganised approach in public health services, but little knowledge or understanding of the infection among patients leads to a lack of recognition of the importance of informing sexual partners. The management of partner(s) was acknowledged as critical for syphilis control in the guidelines and by HCPs, but no document provides comprehensive information about delivering PN. Patient referral was used most commonly; however, interviewees commented that index cases do not discuss their partners easily and PN delivery is further impacted by gender and the socio-cultural context. PN was perceived by HCPs as an exhausting, difficult and challenging process due to poor public understanding of syphilis, absence of practical recommendations and health system limitations.

Conclusion Understanding the state of current policies and practices are important for improving $\mathrm{PN}$ in Chile and Latin America. Lack of clear guidelines and resources, as well as the impact that gender and socio-cultural aspects have on STI risk perceptions, should be considered to strengthen STI control at the population level.

Disclosure No significant relationships.

\section{P248 STI RISK REDUCTION STRATEGIES AMONG INDIVIDUALS WITH MULTIPLE SEX PARTNERS AND PERCEIVED PARTNER NON-MONOGAMY IN THE US}

${ }^{1}$ Casey Copen*, ${ }^{2} J a m i$ Leichliter, ${ }^{1}$ Ian Spicknall, ${ }^{3}$ Sevgi Aral. ${ }^{1}$ Centers for Disease Control and Prevention, Division of STD Prevention, Atlanta, USA; ${ }^{2} C D C$, Atlanta, USA; ${ }^{3} C D C$, Division of STD Prevention, Atlanta, USA

\subsection{6/sextrans-2019-sti.381}

Background Network position contributes to STI risk because of broad sexual network connectivity. Relationship types (i.e., exclusive dating, hook ups) also influence STI risk. Few studies examine how sexual network position and relationship 
types jointly influence individuals' STI risk reduction strategies.

Methods We analyzed data from 2011-2017 National Survey of Family Growth for 5,948 and 5,433 unmarried, non-cohabiting sexually active women and men aged 15-44. To describe sexual network position, we created four dichotomous variables that included both past-year number of opposite-sex sex partners (one, multiple) and perceived partner non-monogamy (PPNM, yes/no). Relationship type was defined as a steady or casual sex partner at last sex. Prevalence ratios were used to assess the relationship between network position and two past-year outcomes: condom use at last sex and STI testing. Results were stratified by relationship type.

Results 54\% and 47\% of women and men aged 15-44 had one partner with no PPNM. $6 \%$ and $4 \%$ had one partner with PPNM, 22\% and 29\% had multiple partners with no PPNM and $17 \%$ and $20 \%$ had multiple partners with PPNM. Men with multiple partners and PPNM had the lowest prevalence of condom use of all four groups $(37.7 \%, \mathrm{CI}$ : 33.5, 44.1 compared with 52-56\%). Moreover, women and men in this group with a steady sex partner had higher prevalence of past-year STI testing if they reported PPNM than if they did not (women: $a P R=1.31$; CI: 1.11, 1.55, men: $a P R=1.47 ; C I: 1.19,1.81)$. This same relationship was not seen for women and men whose last sex was with a casual partner.

Conclusion Individuals' STI risk reduction strategies depend on sexual network position and relationship type. Those with PPNM and a steady sex partner may seek STI testing more frequently. Proxy sexual network measures from national surveys may help target STI prevention and testing interventions. Disclosure No significant relationships.

\section{P249 WHAT WORKS IN PARTNER NOTIFICATION FOR SEXUALLY TRANSMITTED INFECTIONS, INCLUDING HIV? SYSTEMATIC REVIEW AND META-ANALYSIS}

${ }^{1}$ Mweete Nglazi, ${ }^{1}$ Taryn Young, ${ }^{2}$ Catherine Mathews, ${ }^{1}$ Moleen Zunza, ${ }^{3}$ Nicola Low*. 'Stellenbosch University, Centre for Evidence-based Health Care, Cape Town, South Africa; ${ }^{2}$ South African Medical Research Council, Health Systems Research Unit, Cape Town, South Africa; ${ }^{3}$ University of Bern, Institute of Social And Preventive Medicine (ISPM), Bern, Switzerland

\subsection{6/sextrans-2019-sti.382}

Background Partner notification (PN) is a core component of the management of sexually transmitted infections (STI). A range of methods can help to find, test and treat sexual partners of an infected person (index patient). The objective of this review was to assess the effects of different PN strategies in people with STI, including HIV infection.

Methods We conducted this review for the Cochrane STI Review Group. We searched for randomised controlled trials (RCTs) in Cochrane, MEDLINE and EMBASE databases, reference lists and trial registries up to 18 October 2018. Two independent reviewers conducted screening, selection and data extraction. Primary biological outcomes were: repeated infection in index cases with curable STI and new infections in partners of people with HIV. We conducted meta-analysis where appropriate.

Results We included 37 trials from 14 countries in Asia, Australia, Europe, South America, sub-Saharan Africa and USA in patients with bacterial STIs or trichomonas $(n=26$ trials), STI syndromes $(n=5)$, HIV $(n=5)$, mixed STI $(n=1)$. The 37 trials assessed 56 comparisons between PN strategies, with the most common being enhanced patient referral $(n=22)$ and expedited partner therapy (EPT, $n=18$ ) for curable STI and contract referral for $\operatorname{HIV}(n=3)$. For index cases with chlamydia, gonorrhoea or trichomonas as a combined outcome, EPT reduced repeat infection compared with simple patient referral $(\mathrm{n}=6,390$, relative risk $0.71,95 \%$ confidence interval, CI 0.59-0.86, I-squared 0\%) but not compared with enhanced patient referral $(n=1,220$, relative risk 0.96 , 95\% CI $0.6-$ 1.53, I-squared 33\%). For HIV, contract referral, versus simple patient referral, increased the number of partners found infected $(n=1,884$, mean difference 0.15 , 95\% CI $0.06-0.24$, I-squared 0\%).

Conclusion EPT is better than simple patient referral, but not better than enhanced patient referral for curable STI. Many PN strategies have limited evidence. Future trials should strengthen evidence of effectiveness by evaluating existing PN strategies using biological outcomes.

Disclosure No significant relationships.

\section{P250 INTERIM ANALYSIS OF INDIVIDUAL RISK FACTORS, SEXUAL NETWORKS, AND STI RISK IN A MILITARY POPULATION}

${ }^{1}$ Nicholas Magno, ${ }^{2}$ Eric Garges*, ${ }^{2}$ Tzucheg Kao, ${ }^{3}$ Realisha Smith, ${ }^{3}$ Tahaniyat Lalani. 'USUHS, Rockville, USA; ${ }^{2}$ Uniformed Services University of the Health Sciences, Department of Preventive Medicine and Biostatistics, Bethesda, USA; ${ }^{3}$ Henry M. Jackson Foundation, Rockville, USA

\subsection{6/sextrans-2019-sti.383}

Background Sexually transmitted infections (STIs) are a consistent medical concern among military service members, who may experience select rates well above their civilian counterparts. Differences in individual risk behaviors as well as network risk factors are different in military populations which may explain the increased rates of select STIs. We performed a pilot study to validate a STI risk assessment survey tool for future use in the military population.

Methods Naval Medical Center Portsmouth was used to pilot a survey for a larger, egocentric social, and sexual network study in military populations. Study participants $(n=50)$ completed an anonymized computer-assisted survey focusing on STI risk factors, including a sexual partner inventory. We evaluated individual demographics, behavioral risk factors, and sexual partnership formation to include mixing patterns and concurrency. Recent history of STI was self-reported in the survey and validated with the participant's medical record. Fischer's exact test was used if applicable. For partners, data was weighted and Wald Chi-square test was used to assess association between variables.

Results Completion of the survey and sexual inventory by respondents was common. The majority were active duty, enlisted, and male. The survey suggested evidence of concurrency and disassortive mixing by age, race, and active duty status. Respondent $(p=0.0089)$ and sexual partner $(p=0.0401)$ alcohol consumption before sex was common and associated with history of STI. Condom use was inconsistent and less frequent with main/steady partners when compared to casual and anonymous partners.

Conclusion The high completion rate demonstrated in this pilot study support that a military population will complete a detailed STI risk index including sexual partner inventory. Interim data analysis suggests that common individual risk 